ALGEBRA, LOGIC AND NUMBER THEORY

BANACH CENTER PUBLICATIONS, VOLUME 121

INSTITUTE OF MATHEMATICS

POLISH ACADEMY OF SCIENCES

WARSZAWA 2020

\title{
SELECTED METHODS FOR THE CLASSIFICATION OF CUTS, AND THEIR APPLICATIONS
}

\author{
FRANZ-VIKTOR KUHLMANN \\ Institute of Mathematics, University of Szczecin \\ ul. Wielkopolska 15, 70-451 Szczecin, Poland \\ ORCID: 0000-0001-5221-5968Ｅ-mail: franz-viktor.kuhlmann@usz.edu.pl
}

\begin{abstract}
We consider four approaches to the analysis of cuts in ordered abelian groups and ordered fields, their interconnection, and various applications. The notions we discuss are: ball cuts, invariance group, invariance valuation ring, and cut cofinality.
\end{abstract}

1. Introduction. In these notes we deal with (Dedekind) cuts in ordered abelian groups and in ordered fields. (For the definition of the notion of a cut and other notions used in this Introduction, see Section 2 ) We introduce the reader to four approaches to their classification, the links between them, and several applications. The reader should observe that a cut in an ordered field is at the same time a cut in its additive group. Hence even in the case that one is predominantly interested in cuts in ordered fields, up to a certain point their study can be fruitfully carried out in the setting of ordered abelian groups and does not need to make use of the field (or ring) multiplication. At the same time the reader should keep in mind that ordered abelian groups appear in field theory also as the value groups of valuations. In this case, cuts in the value group can for instance be generated by pseudo Cauchy sequences in the valued field. If the field is ordered and its

2010 Mathematics Subject Classification: 06F20, 12J10, 12J15, 12J20, 13J30.

Key words and phrases: ordered abelian group, ordered field, natural valuation, spaces of real places, valued field, cut, invariance group, invariance valuation ring, symmetrically complete, defect, henselization, pseudo Cauchy sequence, power series field, Hahn group.

The final work on this article was supported by Opus grant 2017/25/B/ST1/01815 from the National Science Centre of Poland.

The author would like to thank Katarzyna Kuhlmann, Marcus Tressl and the referee for useful suggestions.

The paper is in final form and no version of it will be published elsewhere. 
valuation is the natural valuation induced by the ordering (see below), then it is essential to study the connection between cuts in the field and induced cuts in the value group.

The first approach to the classification of cuts is to ask whether a cut in an ordered abelian group is the upper or lower edge of a convex subgroup, or of a coset thereof. This has been used and studied more or less explicitly by many authors, and various names have been given to such cuts. We call them ball cuts. They appear implicitly or explicitly, sometimes with surprisingly different definitions, in [14, 15, 16, 17, 41] for the study of cuts in ordered fields, in [51] for the study of cuts in ordered abelian groups, and in several other papers cited in the references. Ball cuts will be introduced and discussed in Section 3

Spaces of $\mathbb{R}$-places (i.e., places with residue fields embeddable in $\mathbb{R}$ ) of ordered fields are not well understood. It is a longstanding open problem which topological spaces appear as spaces of $\mathbb{R}$-places. Recently, ball cuts have been used to study these spaces (cf. [37, 35, 36, 28]). We describe some results in Section 3.2

Two well known deep open problems in positive characteristic are:

1) resolution of singularities in arbitrary dimension,

2) decidability of the field $\mathbb{F}_{p}((t))$ of Laurent series over a finite field.

Both problems are connected with the structure theory of valued function fields of positive characteristic $p$. The main obstruction here is the phenomenon of the defect. Via ramification theory, the study of it can be reduced to the study of purely inseparable extensions and of Galois extensions of degree $p$. Ball cuts are essential for a classification of Galois extensions with nontrivial defect which is introduced in [32] and continued in [4]. It will be discussed in Section 3.3

How "broad" is a given cut? One way to answer this question is to associate to the cut the maximal set of elements that can be added to the cut sets without changing the cut. This set turns out to be a convex subgroup of the ordered group; we call it the (additive) invariance group. This notion was introduced by the author in his thesis [29] in order to handle valued fields with non-archimedean ordered value groups in connection with the model theory of valued fields. Invariance groups were also introduced by M. Tressl in his thesis [47, this time for the study of the model theory of ordered fields (a quick example for Tressl's use of invariance groups is given in Section 4.4. Later, A. Fornasiero and M. Mamino [12] used them in a detailed investigation of cuts of ordered abelian groups, which they then applied to study so-called double ordered monoids. Moreover, they have been implicitly used by several other authors (e.g. by R. Rolland in [44]), or even explicitly defined under different names (e.g. by F. Wehrung in [51], and by D. Kijima and M. Nishi in [24]). A main link to ball cuts is the fact that invariance groups can help to identify them (see Theorem 4.3 below). We will discuss invariance groups in Section 4.

In mathematics, objects that are maximal with respect to a certain property are often of particular interest. In valuation theory, this is so for maximal valued fields, which have the property that every proper extension will necessarily enlarge value group or residue field. Ordered abelian groups and fields carry natural valuations which are canonically derived from their ordering. In Section 4.2 we describe a characterization of 
certain ordered fields maximal with respect to their natural valuation, given by Kijima and Nishi; it makes essential use of invariance groups. Further, we discuss a generalization of this result, due to H.-J. Hüper, to the case of valuations whose valuation rings are convex under the given ordering.

In Section 4.3 we discuss an analogue for the case of ordered abelian groups. We present the Cohen-Goffman Theorem and a related result by P. Ehrlich, which both (implicitly) use invariance groups.

The author's attention was drawn to the importance of invariance groups in the study of ordered fields by a question of J. Madden. During the Special Semester in Real Algebraic Geometry and Ordered Structures, Baton Rouge 1996, Madden showed him the definition of what we now call the invariance valuation ring and asked for the meaning of it. The answer to his question was first given in the manuscript [30]. Again, the invariance valuation ring was independently introduced and applied by M. Tressl (see [47, 48, 49]). The construction of invariance valuation rings appears already in Rolland's paper [44, but not in full generality.

Looking at a cut in an ordered field $K$, one may ask whether it originates in some way from a cut in the residue field of $K$ with respect to some real place. That is, one would like to know whether the cut can be translated into some "normal position" such that for some convex valuation ring $\mathcal{O}$ of $K$ with maximal ideal $\mathcal{M}$, it induces a (Dedekind) cut in the ordered residue field $\mathcal{O} / \mathcal{M}$ via the residue map. If so, one would like to determine how this translation can be done. The invariance valuation ring is a key tool to answer these questions (see Section 5.1.

Further, in the paper 22 F. Jahnke, P. Simon and E. Walsberg introduce certain invariance valuation rings to exhibit definable valuations in ordered fields which are not dense in their real closures. The details will be discussed in Section 5.2 .

A remark by M. Marshall made it clear to the author of these notes that some of his earlier results were actually a special case of a more general setting which we will now sketch. Every ordered field $K$ has a natural valuation $v$, whose residue field is an archimedean ordered field; its valuation $\operatorname{ring} \mathcal{O}_{v}$ is the smallest of all convex valuation rings of $K$. Then every convex subgroup of the ordered additive group of $K$ is an $\mathcal{O}_{v}$-module.

In the present paper, we use the additive Krull notation for valuations, i.e., the ultrametric triangle law reads $v(a+b) \geq \min \{v(a), v(b)\}$ and the value group is an additively written ordered abelian group whose nonnegative elements are precisely the values of the nonzero elements of $\mathcal{O}_{v}$. In this notation, the map

$$
M \mapsto(v K \backslash v M, v M),
$$

where $v K$ is the value group of $(K, v)$ and $v M:=\{v a \mid 0 \neq a \in M\}$, is a bijection between the convex subgroups $M$ of $K$ and the cuts in the value group $v K$. This holds more generally for any (Krull) valuation $v$ of an arbitrary field $K$ and the set of all $\mathcal{O}_{v}$-modules $M \subseteq K$. Information about $M$ can be read off from the invariance group of the cut $(v K \backslash v M, v M)$. One can also define the invariance valuation ring of an $\mathcal{O}_{v}$-module. The invariance valuation ring of a cut can then be understood as the invariance valuation ring of the invariance group of the cut. 
Tressl introduced the author to the definition and main properties of the multiplicative invariance group of a cut in an ordered field, that is, the invariance group of the cut taken in the multiplicative group of the field. For its properties, see [34, where a detailed study of ball cuts, invariance groups and invariance valuation rings is presented. Detailed studies of cuts using these concepts appear also in Tressl's papers [47, 48, 49] and T. Güldenberg's thesis [19].

After ball cuts, invariance group and invariance valuation ring, the fourth approach to the study of Dedekind cuts is to consider the pair of cardinal numbers $(\kappa, \lambda)$ where $\kappa$ is the cofinality of the lower cut set and $\lambda$ is the coinitiality of the upper cut set. Recall that the coinitiality of a linearly ordered set is the cofinality of this set under the reversed ordering. Recall further that cofinalities and coinitialities of ordered sets are regular cardinals. We call $(\kappa, \lambda)$ the cofinality of the cut; also the name character has been used in the literature.

In his groundbreaking and comprehensive work, F. Hausdorff constructs for any given collection of cofinalities $(\kappa, \lambda)$, which satisfy some necessary conditions, a totally ordered set where this collection is exactly the set of cofinalities of the cuts appearing in this ordering. One aim of the already cited paper [44] of Rolland is to construct ordered fields which realize a prescribed set of cut cofinalities.

A much studied property of ordered abelian groups or fields is that of being an $\eta_{\alpha}$-set, which is equivalent to the absence of cuts of cofinality $(\kappa, \lambda)$ with both $\kappa$ and $\lambda$ smaller than $\aleph_{\alpha}$. We discuss a characterization of such ordered abelian groups and fields, due to N. Alling, in Section 6.1

In Section 6.2, we present some work of N. Yu. Galanova and G. G. Pestov which involves cut cofinalities and ball cuts.

More recently, a new aspect of cut cofinalities has been discovered. Transferring the concept of spherical completeness from ultrametric spaces to other spaces equipped with distances or topologies, the authors of [38] asked the question whether there are ordered fields, apart from the reals themselves, in which every chain of closed bounded intervals has a nonempty intersection. This happens exactly when all appearing cut cofinalities $(\kappa, \lambda)$ satisfy $\kappa \neq \lambda$. The positive answer to the question was first given by S. Shelah in the paper [45]. In joint work with Shelah and K. Kuhlmann, the author of these notes gave an alternative construction and a complete characterization of such fields in [39]. We will discuss some details in Section 6.3

These notes are not intended to be a comprehensive survey on the general theory of cuts. However, the author hopes that they will initiate discussion and feedback so that more comprehensive information can be gathered and later be put together in a monograph on cuts.

2. Notation and preliminaries. For general background from valuation theory, we recommend [10]. For background on ordered fields, see [40, 42].

2.1. Cuts. Take any ordered set $(S,<$ ) (by "ordered", we will always mean "totally ordered"). If $S_{1}, S_{2}$ are nonempty subsets of $S$ and $a \in S$, we will write $a<S_{2}$ if $a<b$ for all $b \in S_{2}$, and we will write $S_{1}<S_{2}$ if $a<S_{2}$ for all $a \in S_{1}$. 
A subset $S^{\prime}$ of $S$ is called convex in $(S,<)$ if for every two elements $a, b \in S^{\prime}$ and every $c \in S$ such that $a \leq c \leq b$, it follows that $c \in S^{\prime}$. A subset $S_{1}$ of $S$ is an initial segment of $S$ if for every $a \in S_{1}$ and every $c \in S$ with $c \leq a$, it follows that $c \in S_{1}$. Symmetrically, $S_{2}$ is a final segment of $S$ if for every $a \in S_{2}$ and every $c \in S$ with $c \geq a$, it follows that $c \in S_{2}$. Note that $S_{1}$ is an initial segment of $S$ if and only if $S_{1}$ is convex and $S_{1}<S \backslash S_{1}$. Note also that $\emptyset<S$ and $S<\emptyset$ by definition; so $\emptyset$ is an initial segment as well as a final segment of $S$.

If $S_{1} \subseteq S$ and $S_{2} \subseteq S$ are such that $S_{1}<S_{2}$ and $S=S_{1} \cup S_{2}$, then we will call $\left(S_{1}, S_{2}\right)$ a cut in $S$. Then $S_{1}$ is an initial segment of $S, S_{2}$ is a final segment of $S$, and the intersection of $S_{1}$ and $S_{2}$ is empty. We write $\Lambda^{L}=S_{1}, \Lambda^{R}=S_{2}$, and

$$
\Lambda=\left(\Lambda^{L}, \Lambda^{R}\right) \text {. }
$$

A cut $\left(\Lambda^{L}, \Lambda^{R}\right)$ with $\Lambda^{L} \neq \emptyset$ and $\Lambda^{R} \neq \emptyset$ is called a Dedekind cut. If $\Lambda$ is a cut in $S$, $(T,<)$ is an extension of $(S,<)$ and $a \in T$ is such that $\Lambda^{L} \leq a \leq \Lambda^{R}$, then we will say that a realizes $\Lambda$ (in $T)$.

For any subset $M \subseteq S$, we let $M^{+}$denote the cut

$$
M^{+}=(\{s \in S \mid \exists m \in M: s \leq m\},\{s \in S \mid s>M\}) .
$$

That is, if $M^{+}=\left(\Lambda^{L}, \Lambda^{R}\right)$ then $\Lambda^{L}$ is the least initial segment of $S$ which contains $M$, and $\Lambda^{R}$ is the largest final segment having empty intersection with $M$. If $M=\emptyset$ then $\Lambda^{L}=\emptyset$ and $\Lambda^{R}=M$, and if $M=S$, then $\Lambda^{L}=M$ and $\Lambda^{R}=\emptyset$. Symmetrically, we set

$$
M^{-}=(\{s \in S \mid s<M\},\{s \in S \mid \exists m \in M: s \geq m\}) .
$$

That is, if $M^{-}=\left(\Lambda^{L}, \Lambda^{R}\right)$ then $\Lambda^{L}$ is the largest initial segment having empty intersection with $M$, and $\Lambda^{R}$ is the least final segment of $S$ which contains $M$. If $M=\emptyset$ then $\Lambda^{L}=M$ and $\Lambda^{R}=\emptyset$, and if $M=S$, then $\Lambda^{L}=\emptyset$ and $\Lambda^{R}=M$.

If $M=\{a\}$, we will write $a^{+}$instead of $\{a\}^{+}$and $a^{-}$instead of $\{a\}^{-}$. These two cuts are called principal. Hence if $M$ has a largest element $a$, then $M^{+}=a^{+}$is principal, and if $M$ has a smallest element $a$, then $M^{-}=a^{-}$is principal. The cut $\left(\Lambda^{L}, \Lambda^{R}\right)$ is principal if and only if $\Lambda^{L}$ has a largest element or $\Lambda^{R}$ has a smallest element. In the literature, a principal cut is also called realized or filled, a non-principal cut is called a gap, and a cut for which $\Lambda^{L}$ has a largest element and $\Lambda^{R}$ has a smallest element is called a jump. In [8], Ehrlich calls a cut $\left(\Lambda^{L}, \Lambda^{R}\right)$ continuous if $\Lambda^{L}$ is principal but not a jump.

2.2. Valuation theory. Take an ordered abelian group $G$. Two elements $a, b$ are $\operatorname{archi}$ medean equivalent if there is some $n \in \mathbb{N}$ such that $n|a| \geq|b|$ and $n|b| \geq|a|$. The equivalence class of $a$ is called archimedean class of $a$ and is denoted by $[a]$. The set $\{[a] \mid 0 \neq a \in G\}$ is totally ordered by setting $[a]<[b]$ if and only if $|a|>n|b|$ for all $n \in \mathbb{N}$. Then the class of 0 is the largest element in the set, and it only contains the element 0 . The map $v: a \mapsto v a:=[a]$ is the natural valuation of $G$. It satisfies the triangle inequality $v(a+b) \geq \min \{v a, v b\}$ and $v(-a)=v a$. We call $\{v a \mid 0 \neq g \in G\}$ the value set of $G$ (under $v$ ).

If $G$ is the additive group of an ordered field $K$, then by setting $[a]+[b]:=[a b]$ we obtain an addition on the set of archimedean classes that is compatible with the ordering, and the natural valuation becomes a field (Krull) valuation. 
Take any extension $(L \mid K, v)$ of valued fields, that is, an extension $L \mid K$ of fields and a valuation $v$ on $L$. By $v L$ and $v K$ we denote the value groups of $v$ on $L$ and on $K$, and by $L v$ and $K v$ the residue fields of $v$ on $L$ and on $K$, respectively. Similarly, $v z$ and $z v$ denote the value and the residue of an element $z$ under $v$.

A valued field $(K, v)$ is called henselian if the extension of $v$ to every algebraic extension field $L$ of $K$ is unique, or equivalently, $(K, v)$ satisfies Hensel's Lemma. A henselization of $(K, v)$ is an algebraic extension of $(K, v)$ which is henselian and can be embedded over $K$ in every other henselian extension field of $(K, v)$. Henselizations exist and are unique up to valuation preserving isomorphism over $K$. Therefore, we will speak of the henselization of $(K, v)$ and denote it by $K^{h}$.

Assume that $L \mid K$ is finite and the extension of $v$ from $K$ to $L$ is unique. Then the lemma of Ostrowski says that

$$
[L: K]=p^{\nu} \cdot(v L: v K) \cdot[L v: K v] \quad \text { with } \nu \geq 0
$$

where $p$ is the characteristic exponent of $K v$, that is, $p=$ char $K v$ if this is positive, and $p=1$ otherwise. The factor $\mathrm{d}=p^{\nu}$ is called the defect of the extension $(L \mid K, v)$. If $\mathrm{d}=1$, then we call $(L \mid K, v)$ a defectless extension; otherwise, we call it a defect extension. Note that $(L \mid K, v)$ is always defectless if char $K v=0$.

We call a henselian field $(K, v)$ a defectless field if every finite extension of $(K, v)$ is defectless. An arbitrary field is called a defectless field if its henselization is defectless.

The extension $(L \mid K, v)$ is immediate if for each $z \in L \backslash K$ there is $c \in K$ such that $v(z-c)>v z$; this holds if and only if the canonical embeddings of $v K$ in $v L$ and of $K v$ in $L v$ are onto.

For $z \in L$, we define

$$
v(z-K):=\{v(z-c) \mid c \in K\} \subseteq v L \cup\{\infty\} .
$$

If $(L \mid K, v)$ is immediate, then $v(z-K)$ is a subset of $v K$ without a maximal element, and even more, it is an initial segment.

Immediate extensions of valued abelian groups can be defined as in the case of valued fields. Valued abelian groups and valued fields are called maximal if they do not admit proper immediate extensions.

2.3. Pseudo Cauchy sequences. A pseudo Cauchy sequence in a valued abelian group or field is a sequence $\left(a_{\nu}\right)_{\nu<\lambda}$ of elements, indexed by a limit ordinal $\lambda$ (which is called the length of the sequence), such that for all $\rho<\sigma<\tau<\lambda$,

$$
v\left(a_{\sigma}-a_{\rho}\right)<v\left(a_{\tau}-a_{\sigma}\right) .
$$

In this case, $v\left(a_{\sigma}-a_{\rho}\right)=v\left(a_{\rho+1}-a_{\rho}\right)$. If $\left(a_{\nu}\right)_{\nu<\lambda}$ is a pseudo Cauchy sequence, then the sequence of values $\left(v\left(a_{\nu+1}-a_{\nu}\right)\right)_{\nu<\lambda}$ is strictly increasing. The set $\{b \mid \forall \nu<\lambda: v(b)>$ $\left.v\left(a_{\nu+1}-a_{\nu}\right)\right\}$ is called the breadth of the sequence $\left(a_{\nu}\right)_{\nu<\lambda}$. An element $a$ (in some valued extension group or field) is a limit of the sequence if $v\left(a-a_{\nu}\right)=v\left(a_{\nu+1}-a_{\nu}\right)$ for all $\nu<\lambda$. If $a$ is a limit of the sequence, then also $a^{\prime}$ is a limit if and only if $a-a^{\prime}$ is an element of the breadth. 
A valued abelian group or field is called spherically complete if it admits a limit for every pseudo Cauchy sequence. If the valued abelian group $G^{\prime}$ is an immediate extension of the valued group $G$, then every element $a \in G^{\prime} \backslash G$ is the limit of a pseudo Cauchy sequence in $G$ that does not have a limit in $G$. Hence, every spherically complete valued abelian group or field is maximal. The converse is also true; in the case of valued fields this is shown by I. Kaplansky in [23], where the theory of pseudo Cauchy sequences (which he calls "pseudo-convergent sequences") is nicely laid out.

If $\alpha$ is an ordinal, then $G$ is called $\alpha$-maximal if every pseudo Cauchy sequence in $G$ of length less than $\aleph_{\alpha}$ has a limit in $G$.

2.4. Hahn products and power series fields. Given a linearly ordered index set $I$ and for every $\gamma \in I$ an arbitrary abelian group $C_{\gamma}$, we define a group called the Hahn product (also called Hahn group), denoted by $\mathbf{H}_{\gamma \in I} C_{\gamma}$. Consider the product $\prod_{\gamma \in I} C_{\gamma}$ and an element $c=\left(c_{\gamma}\right)_{\gamma \in I}$ of this group. Then the support of $c$ is the set $\operatorname{supp} c:=\{\gamma \in I \mid$ $\left.c_{\gamma} \neq 0\right\}$. As a set, the Hahn product is the subset of $\prod_{\gamma \in I} C_{\gamma}$ containing all elements whose support is a well-ordered subset of $I$, that is, every nonempty subset of the support has a minimal element. The Hahn product is a subgroup of the product group. Indeed, the support of the (componentwise) sum of two elements is contained in the union of their supports, and the union of two well-ordered sets is again well-ordered.

The support of every nonzero element $c$ in the Hahn product has a minimal element $\gamma_{0}$. This enables us to define a group valuation by setting $v c=\gamma_{0}$ and $v 0=\infty$; this is called the canonical valuation of the Hahn product $\mathbf{H}_{\gamma \in I} C_{\gamma}$.

If the $C_{\gamma}$ are (not necessarily archimedean) ordered abelian groups, we obtain the ordered Hahn product, also called lexicographic product, where the ordering is defined as follows. Given a nonzero element $c=\left(c_{\gamma}\right)_{\gamma \in I}$, let $\gamma_{0}$ be the minimal element of its support. Then we take $c>0$ if and only if $c_{\gamma_{0}}>0$. If all $C_{\gamma}$ are archimedean ordered, then the canonical valuation of the Hahn product coincides with the natural valuation of the ordered Hahn product. The Hahn Embedding Theorem states that every ordered abelian group $G$ can be embedded in the Hahn product with its set of archimedean classes as index set and all $C_{\gamma}$ equal to the ordered group of real numbers.

Take any ordered abelian group $G$. If $H \varsubsetneqq H^{\prime}$ are convex subgroups of $G$ such that the ordering induced on $H^{\prime} / H$ is archimedean (and hence $H^{\prime} / H$ can be seen as an ordered subgroup of the reals), then $H^{\prime} / H$ is called an archimedean component of $G$. If $G=\mathbf{H}_{\gamma \in I} C_{\gamma}$ and all $C_{\gamma}$ are archimedean ordered, then the $C_{\gamma}$ are precisely the archimedean components of $G$.

Take a field $k$ and an ordered abelian group $G$. Then $k((G)):=\mathbf{H}_{\gamma \in G} k$ is a valued abelian group. Since all supports are well-ordered, a multiplication can be defined as follows: $\left(c_{g}\right)_{g \in G} \cdot\left(c_{g}^{\prime}\right)_{g \in G}=\left(\sum_{h+h^{\prime}=g} c_{h} \cdot c_{h^{\prime}}^{\prime}\right)_{g \in G}$. Then $k((G))$ becomes a valued field, called a power series field. The canonical valuation of the underlying Hahn product makes it a valued field with value group $G$ and residue field $k$.

Under their canonical valuation, all Hahn products and all power series fields are spherically complete and hence maximal. All maximal fields with residue fields of characteristic 0 are power series fields, but for positive residue characteristic this is not true. 
3. Ball cuts. We say that a cut $\Lambda=\left(\Lambda^{L}, \Lambda^{R}\right)$ in an ordered abelian group is a group ${ }^{+}$cut if it is induced by the upper edge of a convex subgroup $H$ of $G$, i.e., if $\Lambda=H^{+}$. We will say that $\Lambda$ is a group ${ }^{-}$-cut if it is induced by the lower edge of a convex subgroup $H$ of $G$, i.e., if $\Lambda=H^{-}$. In both cases, we will call $\Lambda$ a group-cut. Note that $0^{+}$and $0^{-}$are the only principal group-cuts. We call $\Lambda$ a ball ${ }^{+}$-cut (or a ball ${ }^{-}$-cut) if it is induced by the upper edge (or lower edge, respectively) of some coset of a convex subgroup $H$ of $G$, i.e., if it is of the form $(g+H)^{+}$(or $(g+H)^{-}$, respectively) for some $g \in G$. Ball ${ }^{+}$-cuts and ball--cuts are called ball-cuts, and cosets $H+g$ of convex subgroups $H$ are also called balls. Note that all group-cuts are ball-cuts.

Ball cuts are called asymmetric cuts in [14, 15, 16, 17, 41. This name is unfortunate; it may have been chosen by the authors after they observed that there are no cuts in ordered fields that are at the same time a ball ${ }^{-}$-cut and a ball ${ }^{+}$-cut. But the situation is different in ordered abelian groups, as the following example shows. Consider the lexicographic ordering on $\mathbb{Z} \times \mathbb{Z}$. Then $\{(0, m) \mid m \in \mathbb{Z}\}^{+}=\{(1, m) \mid m \in \mathbb{Z}\}^{-}$.

In Tressl's paper [49], the ball ${ }^{+}$-cuts are the cuts with signature 1 , and the ball ${ }^{-}$-cuts are the cuts with signature -1 . All non-ball cuts have signature 0 . Güldenberg also uses signatures in his thesis [19], but defines them in a slightly different way.

3.1. Monoids of cuts. On the set of cuts in an ordered abelian group, addition can be defined in various ways. The two immediately obvious ways to define $\Lambda_{1}+\Lambda_{2}$ are the following:

1) set $\Lambda_{1}+\Lambda_{2}:=\left(\Lambda_{1}^{L}+\Lambda_{2}^{L}\right)^{+}=\left\{\alpha+\beta \mid \alpha \in \Lambda_{1}^{L}, \beta \in \Lambda_{2}^{L}\right\}^{+}$,

2) set $\Lambda_{1}+\Lambda_{2}:=\left(\Lambda_{1}^{R}+\Lambda_{2}^{R}\right)^{-}$.

The two additions are usually not the same, but their properties are very similar. The following fact is easy to prove:

The idempotent elements in these monoids are precisely the group cuts.

Monoids of cuts are studied in [51, [19, [11] and [12]. In the latter paper, the results are used for the intrinsic construction (without the use of embeddings in power series fields) of towers of complements to all (possibly fractional) ideals of the valuation ring in henselian valued fields of residue characteristic 0, and in Kaplansky fields (i.e., valued fields satisfying "Hypothesis A" in [23]) which do not admit proper immediate algebraic extensions. They are also used by N. Alling in [1] for the characterization of $\eta_{\alpha}$ ordered abelian groups and fields (see Section 6.1 below). Alling gives credit to A. H. Clifford [5] for introducing the monoid structure (but it had very probably already been observed before, when Dedekind completions of ordered abelian groups were considered).

3.2. Applications to spaces of $\mathbb{R}$-places. For any formally real (i.e., orderable) field $K$, the question arises which orderings induce the same natural valuations. The places associated with natural valuations are called $\mathbb{R}$-places as their residue fields are archimedean ordered and can thus be embedded in $\mathbb{R}$. We will therefore always assume that the residue field of an $\mathbb{R}$-place is a subfield of $\mathbb{R}$. The above question was answered in [35] for an interesting special case. 
Take a real closed field $R$. There is a one-to-one correspondence between orderings $P$ of $R(X)$ and cuts of $R$ (see [18]). The cut $\Lambda_{P}=\left(\Lambda_{P}^{L}, \Lambda_{P}^{R}\right)$ corresponding to $P$ is given by $\Lambda_{P}^{L}=\left\{a \in R \mid a<_{P} X\right\}$ and $\Lambda_{P}^{R}=\left\{b \in R \mid b>_{P} X\right\}$. Conversely, if $\Lambda$ is a cut in $R$, then the set

$$
P=\left\{f \in R(X) \mid \exists a \in \Lambda^{L} \exists b \in \Lambda^{R} \forall c \in(a, b): f(c) \in \dot{R}^{2}\right\}
$$

is an ordering of $R(X)$ with $\Lambda_{P}=\Lambda$ (here, $\dot{R}=R \backslash\{0\}$ ).

In [35] the following result is proved:

THEOREM 3.1. Two distinct orderings of $R(X)$ induce the same $\mathbb{R}$-place if and only if they correspond to the upper and lower edges of the same ball, that is, there is a convex subgroup $H$ of the additive group of $R$ and $c \in R$ such that one of the places corresponds to $(H+c)^{-}$and the other to $(H+c)^{+}$.

This means that the space of $\mathbb{R}$-places of $R(X)$ is obtained from the line $R$ by identifying the upper and lower edges of balls. If this is done for $R=\mathbb{R}$ then we obtain the circle (up to homeomorphism). But if $R$ is a non-archimedean ordered field, then the structure is much more complex; it may be thought of as an infinite pearl necklace in which every pearl contains a pearl necklace that is similar to the whole necklace. The rich self-similarities of this space have been exhibited in [36 by observing that the transformations $a \mapsto a+c, a \mapsto c a$ and $a \mapsto a^{-1}$ all transform balls into balls.

The following result is also proved in [35]:

THEOREM 3.2. Take an ordering on $R(X)$ which extends the ordering of $R$, and take $v$ to be the natural valuation on $R(X)$ with respect to this ordering. Then $X$ induces in $R$ a cut of the form $(c+H)^{-}$or $(c+H)^{+}$(as in Theorem 3.1 if and only if $v R \varsubsetneqq v R(X)$, and if the former is the case, then $v(X-c)$ is rationally independent over $v R$.

From this theorem we conclude that a cut of $R$ is a ball cut if and only if the natural value group $v R(X)$ of the corresponding ordering on $R(X)$ satisfies $[v R(X): 2 v R(X)]=2$.

In the paper [28] P. Koprowski and K. Kuhlmann consider the more general case of an algebraic function field $F$ of transcendence degree 1 over a real closed field $R$. Choose any smooth projective model of $F$, i.e., a smooth, projective algebraic curve over $R$ with function field $F$. In 226, 27. M. Knebusch shows that the curve consists of finitely many semialgebraic connected components, each of which can be endowed with a cyclic order. In 28, this is used to define cuts in these components; the collection of all of them is taken to be the set of cuts on the curve. The following result is proved:

THEOREM 3.3. The space of all cuts on the curve (endowed with the order topology) is homeomorphic to the space of all orderings on $F$ (endowed with the Harrison topology).

Take any ordering of $F$ and let $v$ denote the natural valuation of $F$ with respect to this ordering. Note that the value group $v R$ of $v$ on $R$ is divisible since $R$ is real closed. Therefore, as $\operatorname{trdeg} F \mid K=1$, there are only two possible cases:

a) $v F=v K$, which implies that $(v F: 2 v F)=1$,

b) $v F=v K \oplus \mathbb{Z} \alpha$ for some $\alpha \in v F \backslash v K$, whence $(v F: 2 v F)=2$. 
By the Baer-Krull Theorem, in the first case there is no other ordering on $F$ that induces the same place as the given one. In the second case there is exactly one other ordering that induces the same place.

Now consider the cut that corresponds to the given ordering according to Theorem 3.3 In analogy to the case of a rational function field discussed above, the authors of [28] call this cut a ball cut if the second case holds. The following argument justifies this definition. Pick any element $X \in F \backslash K$. Then $F \mid K(X)$ is algebraic, thus $v F / v K(X)$ is a torsion group. This implies that case b) holds for $F$ with the given ordering if and only if it holds for $R(X)$ with the restriction of this ordering, as the corresponding natural valuation on $R(X)$ is just the restriction of the natural valuation on $F$. From this one obtains:

Proposition 3.4. The following are equivalent:

1) the cut corresponding to the given ordering on $F$ is a ball cut,

2) for some $X \in F \backslash K$, the cut induced by $X$ in $R$ under the restriction of the ordering to $R(X)$ is a ball cut,

3) for each $X \in F \backslash K$, the cut induced by $X$ in $R$ under the restriction of the ordering to $R(X)$ is a ball cut.

All results above can be obtained in an abstract setting for abstract real curves. However, once we embed the curve in an affine space we obtain a clearer picture. Note that every $n$-dimensional affine space $\mathbb{A}^{n} R$ over $R$ is an ultrametric space with the ultrametric generated by the natural valuation $v$ of $R$. The ultrametric distance between points $\left(x_{1}, \ldots, x_{n}\right)$ and $\left(y_{1}, \ldots, y_{n}\right)$ can be defined and computed as follows:

$$
u\left(\left(x_{1}, \ldots, x_{n}\right),\left(y_{1}, \ldots, y_{n}\right)\right)=\min \left\{v\left(x_{i}-y_{i}\right)\right\}=\frac{1}{2} v\left(\sum\left(x_{i}-y_{i}\right)^{2}\right) .
$$

Therefore we can consider ultrametric balls in $\mathbb{A}^{n} R$. We say that an ultrametric ball $B$ in $\mathbb{A}^{n} R$ cuts a curve $\mathcal{C}$ if $B \cap \mathcal{C} \neq \emptyset$ and $\left(\mathbb{A}^{n} R \backslash B\right) \cap \mathcal{C} \neq \emptyset$. In this case $B$ determines a cut (always more than one) on the curve. In 28 it is shown that such a cut is a ball cut, and the following theorem is proved:

THEOREM 3.5. Every ball cut on a smooth and complete real affine curve in $\mathbb{A}^{n} R$ is induced by some ultrametric ball. If the orderings corresponding to two ball cuts induce the same $\mathbb{R}$-place, then there is an ultrametric ball in $\mathbb{A}^{n} R$ which induces both cuts on the curve.

The converse of the second assertion is not true, a counterexample is given in [28]. The ball mentioned in this assertion can induce more than two cuts on the curve. It is an open question how to determine the pairs of cuts that induce the same $\mathbb{R}$-place.

3.3. Classification of Artin-Schreier defect extensions. An Artin-Schreier extension is a field extension $L \mid K$ of degree $p$ of fields of characteristic $p$ generated by an element $\vartheta$ that satisfies $\vartheta^{p}-\vartheta \in K$. Such an extension has nontrivial defect if and only if it is immediate. In this case, the cut $v(\vartheta-K)^{+}$taken in the divisible hull of $v K$ enables us to distinguish two types of Artin-Schreier defect extensions. We call such an extension dependent if it can be derived by a transformation from a purely inseparable defect extension of degree $p$, and independent otherwise. In [32] the following result is proved: 
THEOREM 3.6. An Artin-Schreier defect extension is independent if and only if the cut $v(\vartheta-K)^{+}$is a group ${ }^{-}$-cut.

This classification of Artin-Schreier defect extensions is important because work by M. Temkin (see e.g. [46]) and by the author indicates that dependent defect appears to be more harmful to the above cited problems than independent defect. In the paper [7, S. D. Cutkosky and O. Piltant give an example of an extension of valued function fields consisting of a tower of two Artin-Schreier defect extensions where so-called strong monomialization fails. As the valuation on this extension is defined by use of so-called generating sequences, it is hard to determine whether the Artin-Schreier defect extensions are dependent or independent. However, Cutkosky, L. Ghezzi and S. ElHitti show that both of them are dependent (see e.g. [9]); this again lends credibility to the hypothesis that dependent defect is the more harmful one.

Moreover, the classification is an important tool in the proof of the following theorem in 32$]$ :

THEOREM 3.7. A valued field of positive characteristic is henselian and defectless if and only if each purely inseparable extension is defectless and the field does not allow any proper immediate algebraic extensions.

This theorem in turn is used in 31 for the construction of an example showing that a certain natural axiom system for the elementary theory of $\mathbb{F}_{p}((t))$ ("henselian defectless valued field of characteristic $p$ with residue field $\mathbb{F}_{p}$ and value group a $\mathbb{Z}$-group") is not complete.

It would be desirable to have an analogue of Theorem 3.7 also in the case of valued fields of mixed characteristic (i.e., valued fields of characteristic 0 with residue fields of positive characteristic). The obvious problem is to find the suitable definition of "dependent", since there are no nontrivial purely inseparable extensions in this case. However, the characterization given in Theorem 3.6 offers a chance for the desired generalization. But the next problem we meet is the fact that while a Galois extension of degree $p$ of a field of characteristic $p$ is an Artin-Schreier extension, a Galois extension of degree $p$ of a field of characteristic 0 is a Kummer extension (provided that the field contains a primitive $p$-th root of unity). This problem is overcome in [4] by using a fact proved in earlier papers of the author of these notes: every Galois defect extension of degree $p$ of a henselian valued field $(K, v)$ of characteristic 0 with residue characteristic $p$ and containing a primitive $p$-th root of unity is generated by an element $\vartheta$ whose minimal polynomial is of the form $X^{p}-X-a+g(X)$ where $v a<0$ and $g$ is a polynomial with coefficients in the valuation ring. Therefore, we can define that such a defect extension is independent if and only if the cut $v(\vartheta-K)^{+}$is a group ${ }^{-}$-cut. We are able to show that these independent defect extensions share important properties with the independent defect extensions in equal characteristic; this shows that our choice for generalizing the definition of "independent" is appropriate.

Another justification for our definition in the mixed characteristic case is obtained from the theory of perfectoid fields, as these allow an exchange of information between the mixed characteristic case and the case of equal positive characteristic. All Galois 
defect extensions of prime degree of perfectoid fields in the equal characteristic case are independent, so the same should hold also in the mixed characteristic case. Indeed, this is proved in [4] even for the larger class of deeply ramified fields in the sense of Section 6.6 of [13, which includes all perfectoid fields. In mixed characteristic as well as in equal characteristic, all Galois defect extensions of prime degree of deeply ramified fields are independent. This nourishes the hope that important theorems that have been proved for defectless fields can also be proved for deeply ramified fields (and other classes of fields closely related to them and introduced in [4]).

On the other hand, we are still only able to prove a partial analogue of Theorem 3.7 It is a slightly more general version of the following assertion: Every deeply ramified field that does not allow any proper immediate algebraic extensions is perfect, henselian and defectless. So far, it is not known whether there is an exact analogue in mixed characteristic of the property of being inseparably defectless.

3.4. Approximation of elements in henselizations. Complete valued fields of rank 1 (i.e., with archimedean ordered value group) are henselian, but for valuations $v$ of arbitrary rank this does not hold in general. However, there is a connection between Hensel's Lemma and completions, but these completions have to be taken for residue fields of suitable coarsenings of $v$. This connection was worked out by P. Ribenboim in [43. who used distinguished pseudo Cauchy sequences to characterize the so-called stepwise complete fields; it had been shown by W. Krull that these fields are henselian.

Take any immediate extension $(L \mid K, v)$ of valued fields and $z \in L \backslash K$. We call $z$ weakly distinguished over $K$ if $v(z-K)^{+}$is a ball ${ }^{+}$-cut, and we call $z$ distinguished over $K$ if it is a group ${ }^{+}$-cut. The latter name is chosen since distinguished elements are limits of distinguished pseudo Cauchy sequences.

Now take an arbitrary valued field $(K, v)$ and extend its valuation $v$ to its algebraic closure $\tilde{K}$. Then $\tilde{K}$ contains a unique henselization $K^{h}$ with respect to this extension. The following result is proved in [33], answering a question by B. Teissier. It has recently been reproved by Teissier using methods from algebraic geometry.

TheOREM 3.8. Each element in $K^{h} \backslash K$ is weakly distinguished over $K$.

Note that if $(K, v)$ is of rank 1 , then its henselization lies in its completion and every element $a \in K^{h} \backslash K$ is distinguished over $K\left(\right.$ with $\left.v(a-K)^{+}=(v K)^{+}\right)$.

By " $\alpha>v(a-K)$ " we mean $\alpha>v(a-c)$ for all $c \in K$. Theorem 3.8 is used in 33] to prove the following result:

TheOREM 3.9. Take $z \in \tilde{K} \backslash K$ such that

$$
v(a-z)>v(a-K)
$$

for some $a \in K^{h}$. Then $K^{h}$ and $K(z)$ are not linearly disjoint over $K$, that is,

$$
\left[K^{h}(z): K^{h}\right]<[K(z): K]
$$

and in particular, $K(z) \mid K$ is not purely inseparable.

This theorem has a crucial application in 32 to the classification of Artin-Schreier defect extensions which we discussed in the previous section. The classification was 
originally obtained in [29] under the additional assumption that the fields in question are henselian. With the help of Theorem 3.9 this assumption can be dropped, and so the classification becomes available for valued function fields.

4. The invariance group. For every cut $\Lambda$ in an ordered abelian group $G$, we define

$$
\mathcal{G}(\Lambda):=\left\{g \in G \mid \Lambda^{L}+g=\Lambda^{L}\right\}
$$

and call it the invariance group of $\Lambda$; other authors (e.g. Ehrlich in [8], following Kijima and Nishi [24]) call it the breadth of the cut $\Lambda$. Note that $\Lambda^{L}+g=\Lambda^{L}$ is equivalent to $\Lambda^{R}+g=\Lambda^{R}$.

The proof of the following facts is straightforward (see e.g. [34]).

Lemma 4.1. Take an ordered abelian group $G$ and a Dedekind cut $\Lambda$ in $G$. Then $\mathcal{G}(\Lambda)$ is a convex subgroup of $G$, and $G$ is the disjoint union of the three convex subsets $\Lambda^{L}-\Lambda^{R}$, $\mathcal{G}(\Lambda)$ and $\Lambda^{R}-\Lambda^{L}$, with

$$
\Lambda^{L}-\Lambda^{R}<\mathcal{G}(\Lambda)<\Lambda^{R}-\Lambda^{L} .
$$

COROllary 4.2. The invariance group of $\Lambda$ is trivial if and only if

$$
\Lambda^{R}-\Lambda^{L}=G^{>0} .
$$

The following theorem is proved in [34, but has also been stated (more or less explicitly) by other authors:

THEOREM 4.3. A cut $\Lambda$ in an ordered abelian group is a ball cut if and only if it is the upper or lower edge of a coset of its invariance group, i.e., if there is some $g \in G$ such that $\Lambda=(g+\mathcal{G}(\Lambda))^{+}$or $\Lambda=(g+\mathcal{G}(\Lambda))^{-}$.

REMARK 4.4. R. Baer [3] introduced the notion eigentlicher Schnitt, that is, a Dedekind cut $\Lambda$ in an ordered abelian group $G$ such that for every positive $g \in G$ there are $a \in \Lambda^{L}$ and $b \in \Lambda^{R}$ such that $b-a<g$. By Lemma 4.1 this condition is equivalent to the invariance group of $G$ being trivial. Ehrlich [8] calls them Veronese cuts, and Galanova and Pestov call them fundamental cuts. The non-principal cuts with trivial invariance groups are called dense in Tressl's papers [48, 49].

Several authors, e.g. Rolland in [44] and Wehrung in [51], work with the ball ${ }^{+}$-cuts $\mathcal{G}(\Lambda)^{+}$rather than the invariance groups themselves. The set of all of these cuts in an ordered abelian group $G$ coincides with the set of cuts $H^{+}$where $H$ runs through all (proper) convex subgroups of $G$. (Note that $G$ itself is the invariance group of the two cuts $(G, \emptyset)$ and $(\emptyset, G)$, which are not Dedekind cuts.)

If $\Lambda$ is a cut in an ordered field $K$ and is positive (i.e., $0 \in \Lambda^{L}$ ), then it is also a cut in the ordered abelian multiplicative group of positive elements of $K$. Its invariance group there is called the multiplicative invariance group of $\Lambda$, and we denote it by $\mathcal{G}^{\times}(\Lambda)$.

4.1. Invariance group and pseudo Cauchy sequences. From what we have said about immediate extensions and pseudo Cauchy sequences in Section 2.3. ordered abelian groups or fields that are maximal with respect to their natural valuation contain limits for all pseudo Cauchy sequences. This is why several authors employ pseudo Cauchy sequences to study and to characterize such ordered abelian groups or fields. Certain 
cuts can induce, or be induced by, pseudo Cauchy sequences. For example, if $\left(a_{\nu}\right)_{\nu<\lambda}$ is a pseudo Cauchy sequences which is also strictly increasing, then it is cofinal in the lower cut set of the cut $\Lambda=\left\{a_{\nu} \mid \nu<\lambda\right\}^{+}$, and the following holds (cf. [34]):

THEOREM 4.5. The invariance group of $\left\{a_{\nu} \mid \nu<\lambda\right\}^{+}$is equal to the breadth of the pseudo Cauchy sequence $\left(a_{\nu}\right)_{\nu<\lambda}$.

If the pseudo Cauchy sequence lies in an ordered abelian group $G$, then it induces a Cauchy sequence (i.e., a pseudo Cauchy sequence with breadth $\{0\}$ ) in $G / \mathcal{G}(\Lambda)$.

4.2. Ordered fields with maximal natural valuation. Take an ordered field $K$. We will denote the ordered additive group of $K$ by $K_{+}$. In [24], Kijima and Nishi use the invariance group for the following result:

THEOREM 4.6. The following assertions are equivalent:

1) the natural valuation of $K$ is maximal and its residue field is $\mathbb{R}$,

2) for each cut $\Lambda=\left(\Lambda^{L}, \Lambda^{R}\right)$ in $K$, the induced cut $\left(\Lambda^{L} / \mathcal{G}(\Lambda), \Lambda^{R} / \mathcal{G}(\Lambda)\right)$ in the ordered abelian group $K_{+} / \mathcal{G}(\Lambda)$ is principal.

Here, the induced cut is $\left(\left\{a / \mathcal{G}(\Lambda) \mid a \in \Lambda^{L}\right\},\left\{b / \mathcal{G}(\Lambda) \mid b \in \Lambda^{R}\right\}\right)$; note that the two sets are disjoint by the defining property of $\mathcal{G}(\Lambda)$.

This theorem also holds for any ordered abelian group $G$ in place of the ordered field $K$ if we replace "its residue field is $\mathbb{R}$ " by "all of its archimedean components are isomorphic to $\mathbb{R}^{\text {"; }}$ see the next section.

In his thesis [21] Hüper considers ordered fields with arbitrary compatible valuations (i.e., valuations whose valuation ring is convex, or equivalently, contains the valuation ring of the natural valuation). We will cite one of his main results; in its formulation he uses a notion that is derived from Baer's "eigentlicher Schnitt" (see Remark 4.4 without explicitly using invariance groups. But using them as follows puts the result in a wider context:

THEOREM 4.7. Take an ordered field $K$ with a compatible valuation $v$. Then the following assertions are equivalent:

1) the valuation $v$ is maximal,

2) if $H$ is a $\mathcal{O}_{v}$-submodule of $K$ not contained in a larger $\mathcal{O}_{v}$-submodule $H^{\prime}$ such that there is no $\mathcal{O}_{v}$-submodule properly between $H^{\prime}$ and $H$, and if $\Lambda$ is a cut such that $\Lambda / H:=\left(\Lambda^{L} / H, \Lambda^{R} / H\right)$ is a Dedekind cut in $K_{+} / H$ with trivial invariance group, then $\Lambda / H$ is principal.

Let us evaluate this theorem for the case of $v$ being the natural valuation. In this case, condition 2) can be reformulated as follows:

$\left.2^{\prime}\right)$ if $H$ is a convex subgroup of $K$ which is not contained in a larger convex subgroup $H^{\prime}$ such that $H^{\prime} / H$ is archimedean ordered, and if $\Lambda$ is a cut such that $\Lambda / H:=$ $\left(\Lambda^{L} / H, \Lambda^{R} / H\right)$ is a Dedekind cut in $K_{+} / H$ with trivial invariance group, then $\Lambda / H$ is principal.

Condition 2') can be further reformulated and thereby simplified by using the following two facts: 
LEMmA 4.8. Take a Dedekind cut $\Lambda$ in an ordered abelian group $G$ and a proper convex subgroup $H$ of $G$. Then the following assertions hold.

a) $\Lambda / H$ is a Dedekind cut in $G / H$ if and only if $H \subseteq \mathcal{G}(\Lambda)$.

b) $\mathcal{G}(\Lambda / H)=\{0\}$ if and only if $\mathcal{G}(\Lambda) \subseteq H$.

In view of these facts, condition 2) is equivalent to:

$2 ")$ if $H$ is a convex subgroup of $K_{+}$which is not contained in a larger convex subgroup $H^{\prime}$ such that $H^{\prime} / H$ is archimedean ordered, and if $\Lambda$ is a cut with invariance group $H$, then $\Lambda / H$ is principal.

What is the role of the assumption on $H$ in conditions $2^{\prime}$ ) and 2")? If $H^{\prime}$ is a larger convex subgroup of $K_{+}$such that $H^{\prime} / H$ is archimedean ordered, then $H^{\prime} / H$ is an archimedean component of $K_{+}$and therefore isomorphic to the additive group of $K v$. As the theorem does not assume that the latter is equal to $\mathbb{R}, H^{\prime} / H$ may have a non-principal Dedekind cut, which then gives rise to a non-principal Dedekind cut of $G / H$. So if we are only interested in maximality, then we have to take this case into account. However, if we assume in addition to condition 1 ) that $K v$ is equal to $\mathbb{R}$, then all Dedekind cuts in archimedean components are principal, and we can drop the assumption on $H$. This shows that Theorem 4.6 is a consequence of Theorem 4.7

REMARK 4.9. In [25], the authors state that "the notion of maximal ordered fields was first introduced" in earlier papers of theirs, the earliest published in 1987 by Kijimi and Nishi. This statement is correct only as far as it concerns results published in journals, as the work of Hüper shows.

4.3. Archimedean complete ordered abelian groups. An ordered abelian group $(G,<)$ is called archimedean complete (a notion introduced by H. Hahn) if every proper ordered abelian group extension $\left(G^{\prime},<\right)$ of $(G,<)$ introduces new archimedean classes, or in other words, the natural valuation on $\left(G^{\prime},<\right)$ has a larger value set than on $G$. Hence the archimedean complete ordered abelian groups are precisely the ordered abelian groups that are maximal with respect to their natural valuation and whose archimedean components are as large as possible, that is, isomorphic to the additive group of real numbers. Hahn shows in 20] that archimedean complete ordered abelian groups are precisely the ones that admit an order preserving isomorphism onto a so-called Hahn product with all of its archimedean components equal to the additive group of real numbers. (Hahn products are the analogues for ordered abelian groups of the power series fields.)

Archimedean complete ordered abelian groups $G$ are characterized in the paper [6] by L. W. Cohen and C. Goffman as follows:

THEOREM 4.10. An ordered abelian group $(G,<)$ is archimedean complete if and only if for every proper convex subgroup $H$, the ordered factor group $G / H$ is dense and every cut in $G / H$ with trivial invariance group is principal.

Ehrlich revisits this topic in [8]. Relying on the theorem of Cohen and Goffman, Ehrlich proves: 
THEOREM 4.11. An ordered abelian group $(G,<)$ is archimedean complete if and only if for every cut $\Lambda$, the induced cut in $G / \mathcal{G}(\Lambda)$ is principal, but not a jump.

Since ordered fields admit no jumps, this theorem can be seen as an analogue of Theorem 4.6. Ehrlich shows that the induced cut has trivial invariance group; this is a special case of part b) of Lemma 4.8

We recommend Ehrlich's paper [8] for interesting historical remarks and a detailed list of references.

4.4. Model theory of ordered fields with cuts. In [47, 48, 49], Tressl studies the model theory of real closed fields with a fixed cut. Given a model $M$ of an o-minimal extension $T$ of the theory of real closed fields in a language $\mathcal{L}$, he determines the model theoretic properties of $M$ in the language $\mathcal{L}(\mathcal{D})$ where $\mathcal{D}$ is a predicate for the left cut set $\Lambda^{L}$ of a fixed cut $\Lambda$ in $M$. If $\left(M_{1}, \Lambda_{1}^{L}\right)$ and $\left(M_{2}, \Lambda_{2}^{L}\right)$ are two structures obtained in this way, conditions are found for $\left(M_{1}, \Lambda_{1}^{L}\right)$ and $\left(M_{2}, \Lambda_{2}^{L}\right)$ to be elementarily equivalent in the language $\mathcal{L}(\mathcal{D})$ enhanced by parameters from a common elementary substructure of $M$ and $M^{\prime}$. The main result is rather technical in nature, but for special classes of cuts, the situation is much easier. To illustrate this, the following theorem is taken from [49]:

TheOREM 4.12. Let $A \prec M_{1}, M_{2}$ be models of $T$ and let $\Lambda_{1}, \Lambda_{2}$ be non-principal cuts in $M_{1}, M_{2}$ respectively, with trivial invariance groups. Then $\left(M_{1}, \Lambda_{1}^{L}\right) \equiv_{A}\left(M_{2}, \Lambda_{2}^{L}\right)$ if and only if the restrictions of $\Lambda_{1}$ and $\Lambda_{2}$ to $A$ coincide.

5. The invariance valuation ring. The invariance valuation ring of a cut $\Lambda$ in an ordered field $K$ is defined as

$$
\mathcal{O}(\Lambda):=\{b \in K \mid b \mathcal{G}(\Lambda) \subseteq \mathcal{G}(\Lambda)\}
$$

We denote its maximal ideal $\{b \in K \mid b \mathcal{G}(\Lambda) \varsubsetneqq \mathcal{G}(\Lambda)\}$ by $\mathcal{M}(\Lambda)$.

According to Lemma $4.1 \mathcal{G}(\Lambda)$ is a convex subgroup of the ordered additive group $K_{+}$ of $K$, and we have already noted in the Introduction that every convex subgroup of $K_{+}$ is an $\mathcal{O}_{v}$-module, where $v$ denotes the natural valuation. In this way, the above definition becomes a special case of the following notion. Take any valued field $K$ with valuation $\operatorname{ring} \mathcal{O}_{v}$ and an $\mathcal{O}_{v}$-module $M$ in $K$. The invariance valuation ring of an $\mathcal{O}_{v}$-module $M$ in $K$ is defined as

$$
\mathcal{O}(M):=\{b \in K \mid b M \subseteq M\} .
$$

The relation between multiplicative invariance group and invariance valuation ring is determined in [34]. Also Tressl and Güldenberg obtain results on this topic.

5.1. Projecting cuts into residue fields. Take a convex valuation ring $\mathcal{O}$ of an ordered field $K$, with maximal ideal $\mathcal{M}$. Its residue field $\mathcal{O} / \mathcal{M}$ is again an ordered field, with the ordering induced through the residue map. We will say that the cut $\Lambda$ can be projected into the residue field $\mathcal{O} / \mathcal{M}$ if there are elements $a, c \in K$ such that $c>0$ and $c \Lambda+a$ induces a Dedekind cut

$$
\left(\left(\left(c \Lambda^{L}+a\right) \cap \mathcal{O}\right) / \mathcal{M},\left(\left(c \Lambda^{R}+a\right) \cap \mathcal{O}\right) / \mathcal{M}\right)
$$

in $\mathcal{O} / \mathcal{M}$ via the residue map. 
The following theorem shows for which convex valuation rings $\mathcal{O}$ a cut can be projected into the associated residue field. For a proof, see [34].

\section{THEOREM 5.1.}

1) Take any convex valuation ring $\mathcal{O}$ of $(K,<)$. If $\mathcal{O}(\Lambda) \varsubsetneqq \mathcal{O}$, then the cut $\Lambda$ can be projected into the residue field $\mathcal{O} / \mathcal{M}$. If $\mathcal{O} \varsubsetneqq \mathcal{O}(\Lambda)$, then it cannot be projected into $\mathcal{O} / \mathcal{M}$.

2) The cut $\Lambda$ can be projected into $\mathcal{O}(\Lambda) / \mathcal{M}(\Lambda)$ if and only if $(v \mathcal{G}(\Lambda))^{-}$is a ball ${ }^{+}{ }_{-}$cut.

5.2. Definable valuation rings in ordered fields. It is obvious that if a cut (that is, its lower cut set $\Lambda^{L}$ ) is definable in some extension of the language of ordered rings, then so is $\mathcal{G}(\Lambda)$. It then follows that also the invariance valuation ring is definable.

This observation is put to work in [22], where the following is proved:

Proposition 5.2. Take an ordered field $K$ with real closure $R$. If $K$ is not dense in $R$, then $K$ admits a nontrivial valuation definable in the language of ordered rings.

The idea of the proof is as follows. If $K$ is not dense in $R$, then there is an element $r \in R \backslash K$ and a positive element $a \in R$ such that $|r-c|>a$ for all $c \in K$. Since $R \mid K$ is algebraic, the set $K^{>0}$ of positive elements in $K$ is coinitial in $R^{>0}$, so we can choose $a \in K$. If we set $\Lambda^{L}=\{c \in K \mid c<r\}$, then we obtain a cut $\Lambda$ such that $\Lambda^{R}-\Lambda^{L} \varsubsetneqq K^{>0}$. By Corollary 4.2 its invariance group is thus nontrivial. This implies that the invariance valuation ring is not all of $K$, so the associated valuation is nontrivial. Since $r$ lies in a real closure of $K$, the set $\Lambda^{L}=\{c \in K \mid c<r\}$ is definable, and by what we said above, so are the invariance valuation ring and thus also the associated valuation.

The above arguments also prove the following general principle:

If the lower cut set of some Dedekind cut with nontrivial invariance group in an ordered field is definable, then the field admits a nontrivial definable valuation ring.

Similarly, if an $\mathcal{O}_{v}$-module $M$ in a valued field $(K, v)$ is definable, then so is its invariance valuation ring $\mathcal{O}(M)$. If $K \neq M \neq\{0\}$, then $\mathcal{O}(M)$ is a nontrivial valuation ring. This yields the following general principle:

If a proper nontrivial $\mathcal{O}_{v}$-module in a valued field $(K, v)$ is definable, then the field admits a nontrivial definable valuation ring containing $\mathcal{O}_{v}$.

Note that if a cut $\Lambda$ is definable in the value group $v K$ in a suitable language of valued fields, then the $\mathcal{O}_{v}$-module $\left\{a \in K \mid v a \in \Lambda^{R}\right\}$ is also definable, and it is proper and nontrivial if and only if the cut is a Dedekind cut.

6. Cut cofinalities. Recall that by the cofinality of the cut $\Lambda$ we mean the pair $(\kappa, \lambda)$ where $\kappa$ is the cofinality of $\Lambda^{L}$, and $\lambda$ is the coinitiality of $\Lambda^{R}$.

6.1. Ordered abelian groups and fields that are $\eta_{\alpha}$-sets. Take any ordinal $\alpha$. An $\eta_{\alpha}$-set is an ordered set $S$ such that for any two subsets $A \subseteq S$ and $B \subseteq S$ of cardinality less than $\aleph_{\alpha}$ with $A<B$, there is some $s \in S$ such that $A<s<B$. This is equivalent to saying that $S$ does not admit any cuts of cofinality $(\kappa, \lambda)$ where both $\kappa$ and $\lambda$ are smaller than $\aleph_{\alpha}$. In [1], Alling proves: 


\section{THEOREM 6.1.}

a) An ordered abelian group is an $\eta_{\alpha}$-set if and only if it is $\alpha$-maximal, its value set with respect to the natural valuation is an $\eta_{\alpha}$-set, and all of its archimedean components are isomorphic to $\mathbb{Z}$ or $\mathbb{R}$.

b) An ordered field is an $\eta_{\alpha}$-set if and only if it is $\alpha$-maximal, its value group with respect to the natural valuation is an $\eta_{\alpha}$-set, and its residue field is $\mathbb{R}$.

Every $\aleph_{\alpha}$-saturated ordered abelian group or field is an $\eta_{\alpha}$-set. For the converse, the reader may note that divisible ordered abelian groups and real closed fields are o-minimal. This implies that for them the property of being an $\eta_{\alpha}$-set is equivalent to that of being $\aleph_{\alpha}$-saturated. For results on the variety of $\eta_{\alpha}$ ordered abelian groups or fields, for fixed $\alpha$, see [2].

Ball cuts and invariance groups do not appear explicitly in [1] or [2]. But Rolland draws a connection in [44]. He states that an ordered abelian group is an $\eta_{\alpha}$-set if and only if its value set is an $\eta_{\alpha}$-set and for every Dedekind cut $\Lambda$ with nontrivial invariance group $\mathcal{G}(\Lambda)$, the coinitiality of the upper cut set of $\mathcal{G}(\Lambda)^{+}$is not less than $\aleph_{\alpha}$.

6.2. Cuts in ordered power series fields. The papers [14, 15, 16, 17] of Galanova and Pestov are devoted to the study of cuts in power series fields and in restricted power series fields (in the latter, the cardinalities of the supports of the power series are bounded by a given cardinal number). We cite three theorems from [17]. The cardinality of a set $S$ is denoted by $|S|$, and $|S|^{+}$denotes its successor cardinal.

THEOREM 6.2. Take any ordered abelian group $G$. Then all cuts in the power series field $\mathbb{R}((G))$ are ball cuts.

The proof of this theorem in [17] is long and technical. Let us give the sketch of a shorter and more conceptual proof. We write $K=\mathbb{R}((G))$. Every cut $\Lambda$ in $K$ is realized in some ordered field extension $L$ of $K$ (for instance, if $L$ is a $|K|^{+}$-saturated elementary extension of the ordered field $K)$. As a power series field, $K$ is maximal with respect to its natural valuation. Extend $v$ to the natural valuation of $L$. Then it follows that for every $x \in L \backslash K$ there is some $a \in K$ such that $v(x-a)=\max \{v(x-c) \mid c \in K\}$ since otherwise, $x$ would be a limit of some pseudo Cauchy sequence in $K$ without a limit in $K$, contradicting the fact that $K$ is maximal. The value $v(x-a)$ can only be maximal if either $v(x-a) \notin v K$ or there is $d \in K$ such that $v d=v(x-a)$ and $d^{-1}(x-a) v \notin K v$. But the latter cannot be the case: since $K v=\mathbb{R}$ and $v$ on $L$ is a natural valuation, we must have that $L v=K v$. Hence $\gamma:=v(x-a) \notin v K$. We leave it as an exercise to the reader to show that $\Lambda=(a+\{b \in K \mid v b>\gamma\})^{+}$or $\Lambda=(a+\{b \in K \mid v b>\gamma\})^{-}$.

THEOREM 6.3. Take any ordered abelian group $G$ and a cardinal number $\kappa$ such that $\aleph_{0}<\kappa<|G|$. Denote by $\mathbb{R}((G, \kappa))$ the subfield of $\mathbb{R}((G))$ consisting of all power series with support of cardinality less than $\kappa$. Take a non-ball cut in $\mathbb{R}((G, \kappa))$ of cofinality $(\lambda, \lambda)$. Then $\lambda$ is equal to the cofinality of $\kappa$. In particular, if $\kappa$ is regular, then $\lambda=\kappa$.

THEOREM 6.4. Take a non-principal cut $\Lambda$ in some ordered field $K$ with trivial invariance group, and let $(\kappa, \kappa)$ be its cofinality. Then $\kappa$ is equal to the cofinality of $K$. 
In [4], Rolland states the existence of power series fields that admit cuts with preassigned cofinalities $\left(\kappa_{i}, \lambda_{i}\right), i \in I$, where the $\kappa_{i}$ and $\lambda_{i}$ are infinite regular cardinals. The proof he gives is insufficient, but the result also follows from the work we will discuss in the next section. He uses it to show the existence and to (partially) characterize the ordered fields which admit a closed bounded interval and a continuous function which is unbounded on this interval.

6.3. Symmetrically complete ordered abelian groups and fields. A Dedekind cut with cofinality $(\kappa, \lambda)$ is called symmetric if $\kappa=\lambda$, and asymmetric otherwise. Note that the notion of symmetry used by Galanova and Pestov is quite different from the one defined here. However, Pestov states in [41] (without proof or reference) that if a cut in an ordered field is symmetric in their sense (i.e., it is not a ball cut), then it is also symmetric in the sense of the above definition. A proof is given by Galanova in [14]. In 44], Rolland states the same in full generality for non-ball cuts in ordered abelian groups. The statement is correct, but his proof appears to have a serious gap.

A linearly ordered set $(I,<)$ is called symmetrically complete if every symmetric cut in $I$ has cofinality $(1,1)$, i.e., is a jump. In dense linear orderings (and hence in ordered fields) there are no jumps. Consequently, a dense linear ordering is symmetrically complete if and only if all of its cuts are asymmetric.

For example, $\mathbb{Z}$ and $\mathbb{R}$ are symmetrically complete, but $\mathbb{Q}$ is not. In $\mathbb{Z}$ and $\mathbb{R}$, every Dedekind cut is principal; in $\mathbb{Z}$ all of them have cofinality $(1,1)$, and in $\mathbb{R}$ they have cofinalities $\left(1, \aleph_{0}\right)$ and $\left(\aleph_{0}, 1\right)$. In contrast, in $\mathbb{Q}$ the Dedekind cuts have cofinalities $\left(1, \aleph_{0}\right)$, $\left(\aleph_{0}, 1\right)$ and $\left(\aleph_{0}, \aleph_{0}\right)$.

In [39] it is shown that a symmetrically complete ordered abelian group is spherically complete with respect to its natural valuation and hence a Hahn product, with all of its archimedean components isomorphic to $\mathbb{R}$. Similarly, a symmetrically complete ordered field is spherically complete with respect to its natural valuation and hence a power series field, with residue field $\mathbb{R}$. For Hahn products with all of its archimedean components isomorphic to $\mathbb{R}$ the set of all cut cofinalities is computed from the set of all cut cofinalities of the value set of its natural valuation, and a similar computation is done for power series fields with residue field $\mathbb{R}$. Based on this computation, a full characterization of symmetrically complete ordered abelian groups and fields is obtained. We will now cite a selection of the main results from [39].

We call an ordered set strongly symmetrically complete if it is symmetrically complete and does not have any cuts with cofinalities $\left(1, \aleph_{0}\right)$ or $\left(\aleph_{0}, 1\right)$.

THEOREM 6.5. A non-archimedean ordered field is symmetrically complete if and only if it is spherically complete with respect to its natural valuation, has residue field $\mathbb{R}$ and a dense strongly symmetrically complete value group.

A nontrivial densely ordered abelian group is symmetrically complete if and only if it is spherically complete with respect to its natural valuation $v$, has a dense strongly symmetrically complete value set, and all archimedean components are isomorphic to $\mathbb{R}$. It is strongly symmetrically complete if and only if in addition, the value set has uncountable cofinality. 
In particular, it follows that symmetrically complete ordered abelian groups are divisible and symmetrically complete ordered fields are real closed.

Further, it is shown in [39] that every ordered set can be extended to a dense strongly symmetrically complete ordered set with uncountable cofinality. The reader may note that this result is not explicitly stated in Hausdorff's work. The authors of [39] also tried to give a construction that is as short as possible. It turns out that the constructed orderings are themselves lexicographic, as are the orderings on Hahn products and power series fields. Such orderings deserve to be studied in more detail.

Using the above results and the fact that every ordered set is the natural value set of some Hahn product with all components isomorphic to $\mathbb{R}$, and every ordered abelian group is the natural value group of some power series field with residue field $\mathbb{R}$, the following result of [45] is reproved:

THEOREM 6.6. Every ordered field can be embedded in a symmetrically complete ordered field. Every ordered abelian group can be embedded in a symmetrically complete ordered abelian group.

\section{References}

[1] N. L. Alling, On the existence of real-closed fields that are $\eta_{\alpha}$-sets of power $\aleph_{\alpha}$, Trans. Amer. Math. Soc. 103 (1962), 341-352.

[2] N. L. Alling, S. Kuhlmann, On $\eta_{\alpha}$-groups and fields, Order 11 (1994), 85-92.

[3] R. Baer, Dichte, Archimedizität und Starrheit geordneter Körper, Math. Ann. 188 (1970), 165-205.

[4] A. Blaszczok, F.-V. Kuhlmann, Deeply ramified fields, semitame fields, and the classification of defect extensions, arXiv: 1811.04396 .

[5] A. H. Clifford, Completion of semi-continuous ordered commutative semigroups, Duke Math. J. 26 (1959), 41-59.

[6] L. W. Cohen, C. Goffman, On completeness in the sense of Archimedes, Amer. J. Math. 72 (1950), 747-751.

[7] S. D. Cutkosky, O. Piltant, Ramification of valuations, Adv. Math. 183 (2004), 1-79.

[8] P. Ehrlich, Dedekind cuts of Archimedean complete ordered abelian groups, Algebra Universalis 37 (1997), 223-234.

[9] S. ElHitti, L. Ghezzi, Dependent Artin-Schreier defect extensions and strong monomialization, J. Pure Appl. Algebra 220 (2016), 1331-1342.

[10] A. J. Engler, A. Prestel, Valued Fields, Springer Monogr. Math., Springer, Berlin, 2005.

[11] A. Fornasiero, F.-V. Kuhlmann, S. Kuhlmann, Towers of complements to valuation rings and truncation closed embeddings of valued fields, J. Algebra 323 (2010), 574-600.

[12] A. Fornasiero, M. Mamino, Arithmetic of Dedekind cuts of ordered abelian groups, Ann. Pure Appl. Logic 156 (2008), 210-244.

[13] O. Gabber, L. Ramero, Almost Ring Theory, Lecture Notes in Math. 1800, Springer, Berlin, 2003.

[14] N. Yu. Galanova, Symmetry of sections in fields of formal power fields and on the nonstandard real line, Algebra Logika 42 (2003), 26-36; English transl.: Algebra Logic 42 (2003), $14-20$. 
[15] N. Yu. Galanova, Symmetric and asymmetric gaps in some fields of formal power series, Serdica Math. J. 30 (2004), 495-504.

[16] N. Yu. Galanova, An investigation of the fields of bounded formal power series by means of theory of cuts, Acta Appl. Math. 85 (2005), 121-126.

[17] N. Yu. Galanova, G. G. Pestov, Symmetry of cuts in fields of formal power series, Algebra Logika 47 (2008), 174-185; English transl.: Algebra Logic 47 (2008), 100-106.

[18] R. Gilmer, Extension of an order to a simple transcendental extension, in: Ordered Fields and Real Algebraic Geometry, Contemp. Math. 8, Amer. Math. Soc., Providence, 1982, $113-118$.

[19] T. Güldenberg, Elementare Invarianten von Dedekindschnitten angeordneter Körper, Diplomarbeit (MSc thesis), Universität Regensburg, 2004; available at: http://math.usask.ca/fvk/asom3.pdf.

[20] H. Hahn, Über die nichtarchimedischen Grössensysteme, Sitzungsberichte der Kaiserlichen Akademie der Wissenschaften, Wien, Mathematisch-Naturwissenschaftliche Klasse 116 (Abteilung IIa) (1907), 601-655.

[21] H.-J. Hüper, Über ordnungsverträglich bewertete, angeordnete Körper, Dissertation, Univ. München, Munich, 1977.

[22] F. Jahnke, P. Simon, E. Walsberg, dp-minimal valued fields, J. Symb. Log. 82 (2017), 151-165.

[23] I. Kaplansky, Maximal fields with valuations I, Duke Math. J. 9 (1942), 303-321.

[24] D. Kijima, M. Nishi, The pseudo-convergent sets and the cuts of an ordered field, Hiroshima Math. J. 19 (1989), 89-98.

[25] D. Kijima, M. Nishi, M. Sakaibara, Maximal extensions of ordered fields, Hiroshima Math. J. 18 (1988), 485-492.

[26] M. Knebusch, On algebraic curves over real closed fields. I, Math. Z. 150 (1976), 49-70.

[27] M. Knebusch, On algebraic curves over real closed fields. II, Math. Z. 151 (1976), 189-205.

[28] P. Koprowski, K. Kuhlmann, Places, cuts and orderings of function fields, J. Algebra 468 (2016), 253-274.

[29] F.-V. Kuhlmann, Henselian function fields and tame fields, extended version of doctoral thesis, Heidelberg, 1990.

[30] F.-V. Kuhlmann, Invariance valuation ring of cuts in ordered fields, manuscript, Baton Rouge, 1996.

[31] F.-V. Kuhlmann, Elementary properties of power series fields over finite fields, J. Symbolic Logic 66 (2001), 771-791.

[32] F.-V. Kuhlmann, A classification of Artin-Schreier defect extensions and a characterization of defectless fields, Illinois J. Math. 54 (2010), 397-448.

[33] F.-V. Kuhlmann, Approximation of elements in henselizations, Manuscripta Math. 136 (2011), 461-474.

[34] F.-V. Kuhlmann, Invariance group and invariance valuation ring of a cut, in preparation; preliminary version available at http://math.usask.ca/ fvk/CUTS.pdf

[35] F.-V. Kuhlmann, M. Machura, K. Osiak, Metrizability of spaces of $\mathbb{R}$-places of function fields of transcendence degree 1 over real closed fields, Comm. Algebra 39 (2011), 3166-3177.

[36] K. Kuhlmann, The structure of spaces of $\mathbb{R}$-places of rational function fields over real closed fields, Rocky Mountain J. Math. 46 (2016), 533-557.

[37] K. Kuhlmann, F.-V. Kuhlmann, Embedding theorems for spaces of $\mathbb{R}$-places of rational function fields and their products, Fund. Math. 218 (2012), 121-149. 
[38] K. Kuhlmann, F.-V. Kuhlmann, A common generalization of metric, ultrametric and topological fixed point theorems, Forum Math. 27 (2015), 303-327; Correction: Forum Math. 27 (2015), 329-330; corrected version at: http://math.usask.ca/fvk/GENFPTAL.pdf

[39] K. Kuhlmann, F.-V. Kuhlmann, S. Shelah, Symmetrically complete ordered sets, abelian groups, and fields, Israel J. Math. 208 (2015), 261-290.

[40] T. Y. Lam, Orderings, Valuations and Quadratic Forms, CBMS Regional Conf. Ser. in Math. 52, Amer. Math. Soc., Providence, RI, 1983.

[41] G. G. Pestov, On the theory of sections in ordered fields, Sibirsk. Mat. Zh. 42 (2001), 1350-1360; English transl.: Siberian Math. J. 42 (2001), 1123-1131.

[42] A. Prestel, Lectures on Formally Real Fields, Lecture Notes in Math. 1093, Springer, Berlin, 1984.

[43] P. Ribenboim, Théorie des valuations, Les Presses de l'Université de Montréal, 1964.

[44] R. Rolland, Étude des coupures dans les groupes et corps ordonnés, in: Real Algebraic Geometry and Quadratic Forms (Rennes, 1981), Lecture Notes in Math. 959, Springer, Berlin, 1982, 386-405.

[45] S. Shelah, Quite complete real closed fields, Israel J. Math. 142 (2004), 261-272.

[46] M. Temkin, Inseparable local uniformization, J. Algebra 373 (2013), 65-119.

[47] M. Tressl, Dedekind cuts in polynomially bounded, o-minimal expansions of real closed fields, doctoral thesis, Regensburg, 1996.

[48] M. Tressl, Model completeness of o-minimal structures expanded by Dedekind cuts, J. Symbolic Logic 70 (2005), 29-60.

[49] M. Tressl, The elementary theory of Dedekind cuts in polynomially bounded structures, Ann. Pure Appl. Logic 135 (2005), 113-134.

[50] M. Tressl, Pseudo completions and completions in stages of o-minimal structures, Arch. Math. Logic 45 (2006), 983-1009.

[51] F. Wehrung, Monoids of intervals of ordered abelian groups, J. Algebra 182 (1996), 287-328. 